\title{
VALIDATION OF FECAL-BASED METHODS FOR MONITORING NUTRITION AND REPRODUCTION OF MOOSE IN THE GREATER YELLOWSTONE ECOSYSTEM
}

\section{JACOB R. GOHEEN $\uparrow$ BRETT R. JESMER $\uparrow$ UNIVERSITY OF WYOMING $\uparrow$ LARAMIE, WY}

\section{INTRODUCTION}

Understanding the influence of habitat and climate on wildlife nutrition, reproduction and demography is a major goal for natural resource managers and ecologists alike. Although both topdown (i.e., predation and disease) and bottom-up (i.e. habitat and nutrition) forces impact demography, the nutritional condition of an animal is an integration of its environment (Parker et al. 2009) and influences reproduction and survival (Clutton-Brock et al. 1987, Keech et al. 2000, Cook et al. 2004), thus allowing for the identification of limiting factors. Researchers and managers must understand which factors limit population growth before mitigating actions can be taken.

The demography of large herbivores is affected by juvenile survival (i.e., recruitment; Gaillard et al. 2000, Raithel et al. 2007), and natural resource managers often index recruitment by estimating juvenile-adult female ratios via ground or aerial surveys (Czaplewski et al. 1983). Although these ratios may be a robust characterization of recruitment, they are likely not a good measure of reproductive rate in populations where neonates and juveniles are frequently consumed by large carnivores. Additionally, because pregnancy and subsequent survival of juveniles is strongly influenced by maternal condition (Clutton-Brock et al. 1987, Keech et al. 2000, Cook et al. 2004), recruitment rates alone cannot provide inference regarding the mechanism (i.e. relative strengths of top-down vs. bottom-up limitation) driving variation in reproduction and survival of young.

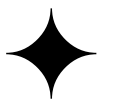

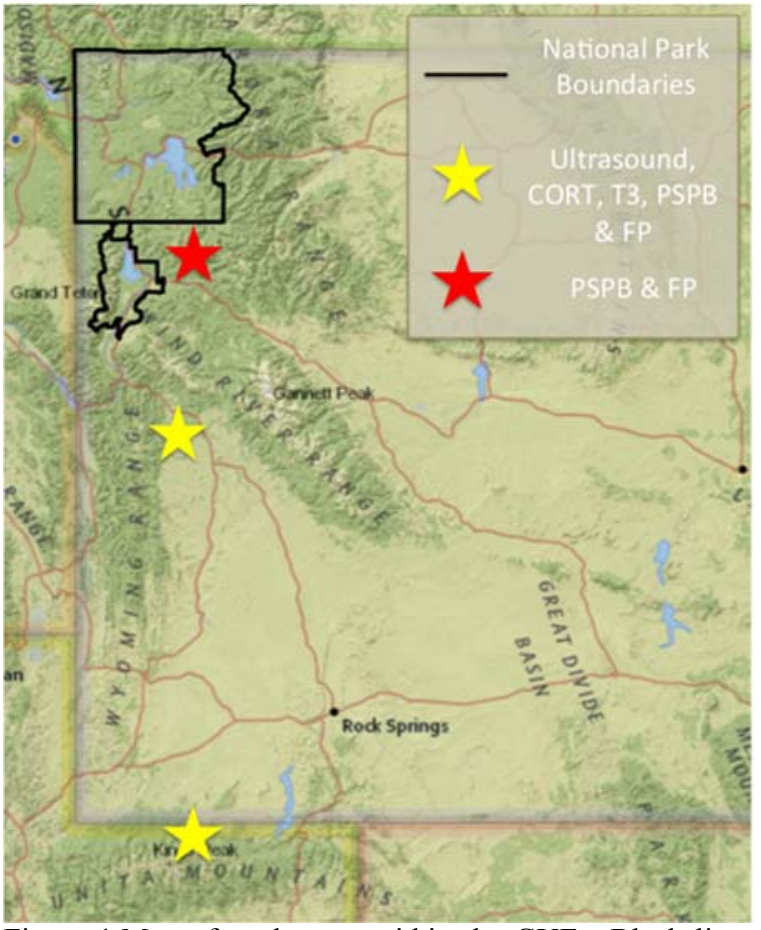

Figure 1.Map of study area within the GYE. Black lines delineate National Park boundaries. Yellow stars indicate sampling areas where ultrasound, GC (CORT), T3, PSPB and fecal progestogen (FP) data were collected. Red stars indicate sampling areas where only PSPB and FP data were collected.

Only recently have non-invasive methods for obtaining in-vivo nutritional data been developed and tested in free-ranging animals. Researchers rely on measures of body condition and reproduction through ultrasonography, body condition scoring, and presence of pregnancy specific protein B (PSPB) in serum to inform them about habitat quality, nutritional 
condition and reproductive rates (Keech et al. 2000, Cook et al. 2004, Monteith et al. 2014). However, these methods are expensive and require the capture and handling of animals, which may be undesirable in many settings. Alternatively, more affordable, noninvasive, fecal-based methods to estimate nutritional condition and pregnancy may be used. The lessprohibitive nature of fecal-based approaches provides researchers and managers with data on nutritional condition and reproduction, and hence the potential to advance our understanding of habitat-demography relationships.

Increasingly, two popular methods for noninvasive (fecal-based) assessment of nutritional condition are the endocrine markers known as glucocorticoids such as cortisol and corticosterone (GC; Wasser et al. 2000) and triiodothryonine (T3; Wasser et al. 2010). GCs provide nutritional support during stressful events, such as unpredictable food shortages or predator escape, by promoting lipid metabolism and glucose production in support of metabolic needs. This adaptive response helps maintain energy balance during periods of restricted caloric intake (McEwen and Wingfield 2003). When reduced caloric intake is chronic, animals rely more heavily on endogenous reserves of energy (Parker et al. 2005), and a continued GC response mobilizes energy for metabolic needs. Due to this dynamic, GCs are expected to increase as nutritional condition declines (e.g., du Dot et al. 2009, Schultner et al. 2013), but this relationship may vary at similar levels of nutritional condition based on forage quality and availability (McEwen and Wingfield 2003, Wikelski and Cooke 2006). Interestingly, the GC response to negative energy balance is expected to have a cascading effect on thyroid function and metabolism (Eales 1988). T3, a prominent thyroid hormone, plays an important role in regulating metabolism and is positively correlated with metabolic rate (Danforth and Burger 1989). T3 is predicted to decrease with declining caloric intake and reduce energy use through its influence on metabolism (Danforth 1984). Thus, fecal measures of GC and T3 provide a potential lens through which nutritional limitation can be studied.

Because GCs rise dramatically when animals are experiencing a negative energy balance (McEwen and Wingfield 2003) and subsequently promote the mobilization of endogenous energy reserves (Porterfield 1997), this endocrine marker consistently relates to the nutritional condition in free-ranging vertebrates (e.g., Kitaysky et al. 1999, du Dot et al. 2009, Schultner et al. 2013). However, there are few examples of studies that quantify the relationship between T3 and the nutritional condition of free- ranging animals, including large herbivores (but see Bahnak et al. 1981, Bishop et al. 2009), with the majority of the literature focused on sudden and unpredictable caloric restriction in laboratory settings (see Eales 1988 for review). In contrast, caloric restriction in large herbivores inhabiting temperate latitudes is predictable (i.e., during the winter season) and this taxonomic group is known to carry over energy reserves between seasons to deal with such caloric bottlenecks (Middleton et al. 2013, Monteith et al. 2013). Therefore, the endocrine responses of large herbivores may be considerably different from the responses to starvation and caloric restriction observed in laboratory settings or in free-ranging animals that do not store substantial energy reserves.

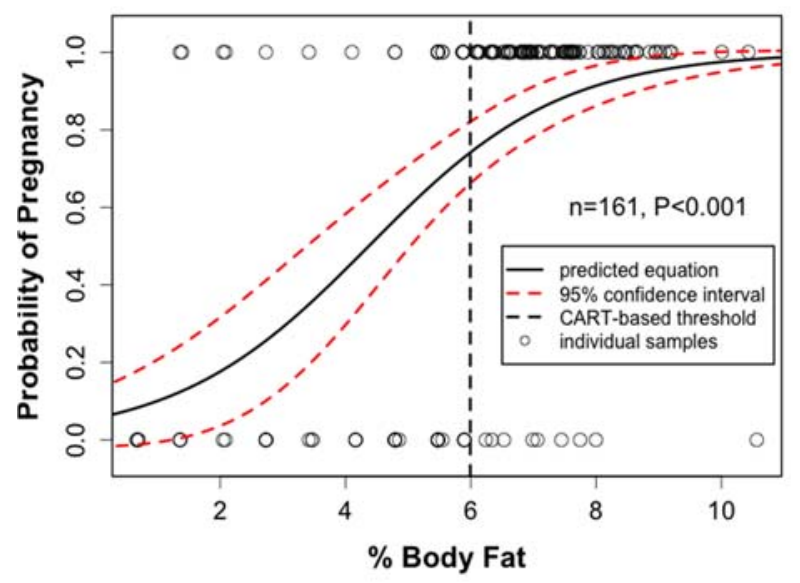

Figure 2. Percent body fat (February) in relation to pregnancy. The vertical, hashed line represents the CARTbased percent body fat threshold for pregnancy, meaning body fat must be typically $>6 \%$ during mating season. Because of such a nutritional threshold, pregnancy rate can be used as a proxy for indexing individual- and populationlevel nutritional condition.

The life-history strategies of large herbivores result in energetic trade-offs between reproduction and survival, where survival is highly conserved (Stearns 1992, Eberhardt 2002). Because of this life history, pregnancy status of individuals and pregnancy rates of populations are indicative of their nutritional condition (e.g., see Figure 2). Progesterone has been used to evaluate pregnancy in mammals for decades, and progestagen (progesterone metabolite) levels found in feces are indicative of pregnancy status (Monfort et al. 1993, Garrott et al. 1998, Cain et al. 2012, Murray et al. 2012). Historically, use of the fecal-based method to accurately determine pregnancy status has been hindered by limited statistical evaluation of progestagen thresholds. Several studies of large herbivores with known pregnancy status based on PSPB analysis use simple visual assessments (Monfort et al. 1993, Cook et al. 2002, Murray et al. 2012) or 
comparisons of pregnant and non-pregnant groups (Garrott et al. 1998, Berger et al. 1999) to determine fecal progestagen thresholds (but see Cain et al. 2012) for a rigorous assessment of fecal progestagen threshold for large herbivores with uncertain pregnancy status). If fecal progestagen thresholds for species can be determined, researchers will be able to perform single-sample pregnancy tests, and thus quantify nutritional condition and reproduction of individuals and populations.

By understanding the relationship between T3, GC, and nutritional condition, non-invasive techniques for evaluating the linkages between habitat, nutritional condition, pregnancy and recruitment rates can be established. By comprehending the extent to which populations are limited by resources while simultaneously quantifying pregnancy and juvenile survival (i.e., calf-cow ratios or recruitment), researchers and managers can assess the relative influences of top-down and bottom-up forces on a population (Bowyer et al. 2005). Herein, we first validate the relationship between $\mathrm{GC}, \mathrm{T} 3$ and nutritional condition, as well as develop a fecal-based pregnancy test for moose in the Greater Yellowstone Ecosystem. Secondly, based on the accuracy of our fecal-based approaches for monitoring nutritional condition and reproduction, we provide suggestions for methodological frameworks and future research. To validate the relationship between GC, T3, nutritional condition and pregnancy, we test the following predictions:

1. GC concentrations will be negatively related to nutritional condition. We expect higher levels of stress, indicated by high concentrations of GC, in individuals in poor nutritional condition.

2. T3 concentrations will be positively related to nutritional condition. We expect higher metabolic rates, indicated by high concentrations of $\mathrm{T} 3$, in individuals in good nutritional condition.

3. Probability of pregnancy will be positively related to the concentration of progestagens found in feces. We expect there to be a clear threshold in fecal progestagen concentration that is indicative of pregnancy.

\section{$\downarrow \quad$ METHODS}

\section{Sampling}

To assess the relationships between GC, T3 and nutritional condition, as well as to develop a fecal progestagen threshold for determining pregnancy status, we used the gold standards of ultrasonography and PSPB analysis to determine the nutritional condition and pregnancy status, respectively, of moose in the GYE and northern Utah (Figure 1). We captured 55 adult, female moose on winter ranges using a handheld net gun fired from helicopter. Each moose was hobbled and blindfolded prior to data collection. To determine the nutritional condition of a subset of moose $(n=36)$, ultrasonography was used to determine the maximum depth of subcutaneous rump fat (Stephenson et al. 1998) and a palpation method was used to develop a body condition score (Cook et al. 2007). Ingesta-free body fat (IFBFAT; percent body fat) was calculated using an extension of Cook et al. (2010) predictive equations (Monteith et al. In Prep). A blood sample $(20 \mathrm{ml})$ was collected via jugular punch and feces were extracted in all moose $(n=55)$. Blood samples were centrifuged and serum was pipetted into a $5 \mathrm{ml}$ cryovials and stored at $-20^{\circ} \mathrm{C}$ until analyzed for PSPB concentration. Fecal samples $(n=55)$ were placed in a plastic bag and stored at $-20^{\circ} \mathrm{C}$ until analyzed for $\mathrm{GC}, \mathrm{T} 3$ and progestagen concentrations.

\section{Measuring PSPB and fecal hormones}

The commercially-available BioPRYN wild assay was used for determination of PSPB concentration of all samples $(n=55)$, and was completed by BioTracking LLC (Moscow, ID). BioPRYN wild is a typical sandwich enzymelinked immunosorbent assay (ELISA) for determination of PSPB levels in serum samples that utilizes horseradish peroxidase labeled antibodies to cause a color change in the sample wells of a 96-well microplate. The assay includes 4 standards run in duplicate on each plate. The standards are halving dilutions from $1 \mathrm{ng} / \mathrm{ml}$ to $0.125 \mathrm{ng} / \mathrm{ml}$. Color development of the assay occurs with the addition of $3,3^{\prime}, 5,5^{\prime},-$ Tetramethylbenzidine. Sulfuric acid is added to stop the reaction. The presence of color development was used to determine pregnancy status. In a subset of individuals $(n=25)$, the optical density for each well was obtained from a plate reader with a filter wavelength of $450 \mathrm{~nm}$ (VersaMax, Molecular Devices, Inc). Simple linear regression was then used to fit an equation to the standards for each plate. The resulting equation was used to calculate a quantitative measure of PSPB concentration in each serum sample.

Pellets from each of 55 moose fecal samples were homogenized, and then freeze-dried. All samples were freeze-dried for 24-48 hours in a Labconco Freeze-Dry system at $-50^{\circ} \mathrm{C}$, and then thoroughly homogenized into a fine powder. Approximately $0.1 \mathrm{~g}$ 
was weighed from each sample, and a pulse-vortex double extraction with $15 \mathrm{~mL} 70 \%$ ethanol was performed. Ethanol extracts were then stored at $-20^{\circ} \mathrm{C}$ until assay. Radioimmunoassays were performed on ethanol extracts at previously validated dilutions for fecal GC and T3 $(n=36)$, and progestagens $(n=55)$, using MP Biomedicals' 125-I corticosterone kit, 125I Total T3 kit \& an in-house 3-H progesterone assay, respectively. The Center for Conservation Biology at the University of Washington (Seattle, WA) conducted GC, T3, and progestagen assays for 36 fecal samples, with the remaining 19 progestagen assays performed by the Smithsonian Conservation Biology Institute (Front Royal, VI). Levels of fecal hormones were then reported as ng per gram of dried feces. All hormone extracts were run in duplicate in each assay, and only those with intra-assay variation $(\% \mathrm{CV})$ below $10 \%$ were accepted, and controls were included in each assay to track inter-assay variation.

\section{Validation of nutritional, GC and T3 relationships}

For $\mathrm{GC}$ and $\mathrm{T} 3$ to be a biologically meaningful metric of nutritional state (i.e., malnutrition) we must understand how these hormones relate to the nutritional condition of animals. To determine the relationship between GC, T3 and nutritional condition (IFBFAT; percent body fat) simple linear regression was applied (Kutner and Nachtsheim 2004). In these models we treat nutritional condition, as determined by a gold standard (i.e., ultrasonography and BCS techniques), as the dependent variable and fecal GC and T3 concentrations as the independent measure. Predicted equations, 95\% confidence intervals and 95\% prediction intervals are presented (Kutner and Nachtsheim 2004).

\section{Fecal progestagen as threshold for pregnancy}

To develop a fecal progestagen threshold from which pregnancy can be determined for any given fecal sample, we applied a classification and regression tree (CART; Breiman 1984) analysis. CART is especially appropriate for our purposes because the classification component is designed to produce discriminant values of independent variables in the process of predicting values associated with the dependent variable (Breiman 1984). In this model we treat pregnancy status, as determined by a gold standard (i.e., ELISA measures of PSPB), as the dependent variable and fecal progestagen concentration as the independent measure. We also present the results of a logistic regression, including the predicted equation and associated $95 \%$ confidence interval, as a visual representation of the data.

\section{$\uparrow \quad$ RESULTS}

\section{Validation of nutritional, GC and T3 relationships}

Fecal GCs were weakly related to nutritional condition (Figure 3, $\mathrm{n}=36, \mathrm{P}=0.16$ ), but suggest that individuals in poor nutritional condition are characterized by high CG levels. This model possessed little predictive power, as indicated by wide prediction intervals (Figure 3). Conversely, fecal T3 was more strongly related to nutritional condition $(\mathrm{n}=36, \mathrm{P}=0.008)$. However, the relationship is negative (Figure 4), indicating that individuals in poor nutritional condition had relatively high metabolic rates, which is in contrast to our prediction. Similar to the relationship between GC and nutritional condition, the T3 model lacked predictive power (Figure 4). Although neither fecal GC nor T3 possessed predictive power, these hormonal markers may still serve as an index to nutritional condition when comparing populations or subpopulations (see Discussion).

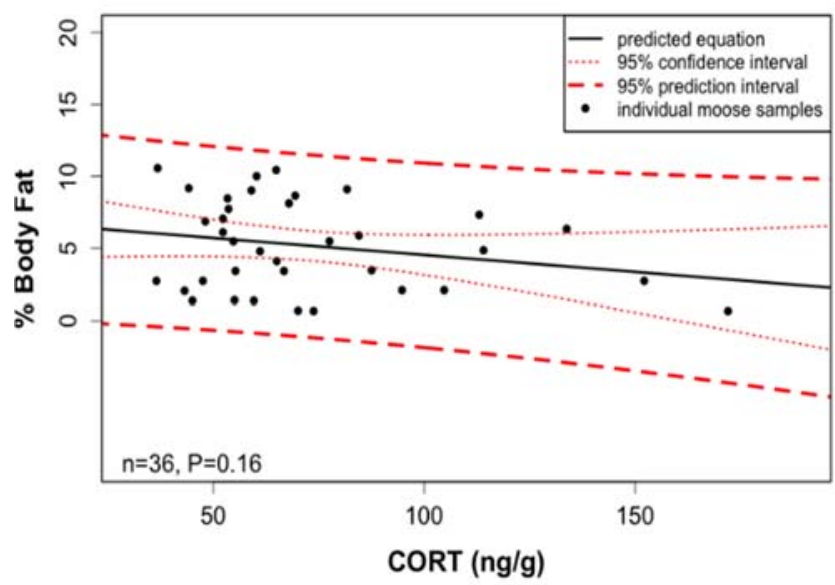

Figure 3. Relationship between percent body fat and fecal GC (CORT) concentrations.

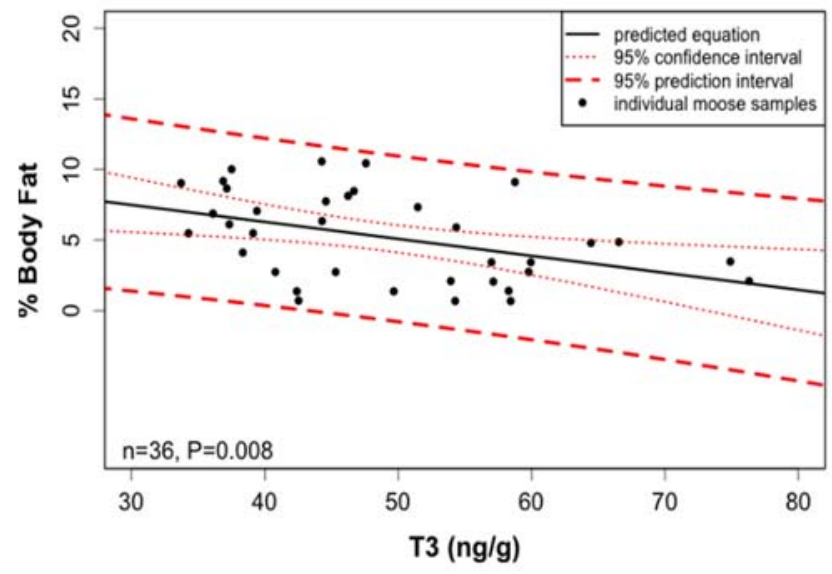

Figure 4. Relationship between percent body fat and fecal T3 concentrations. 


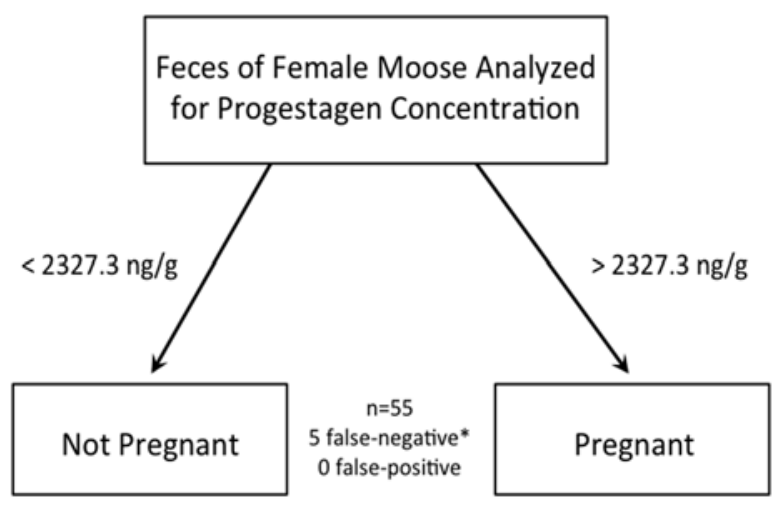

Figure 5. Graphical representation of the classification tree used to calculate the fecal progestagen threshold for determination of pregnancy status. * See Figure 6 and discussion for additional dialogue regarding the 5 misclassified (false-negative) individuals.

\section{Fecal progestagen threshold for pregnancy determination}

CART revealed a fecal progestragen threshold of $2327.3 \mathrm{ng} / \mathrm{g}$ (Figure 5). This threshold resulted in a 91\% (50/55) accurate assessment of pregnancy based on fecal progestagens, indicating a clear fecal progestagen threshold for determination of pregnancy status. All five misclassifications were false-negatives (Figure 6), suggesting possible fetal reabsorption (Cook et al. 2002; see discussion). Logistic regression indicated a strong relationship between fecal progestagen concentration and pregnancy status $(\mathrm{n}=55, \mathrm{P}=<0.001)$. Even though a threshold of $2327.3 \mathrm{ng} / \mathrm{g}$ resulted in the misclassification of 5 individuals, quantitative ELISA assessment of PSPB may support the notion that some or all of our 5 false-negatives are indeed cases of fetal reabsorption, resulting in an increase in fecal-based pregnancy detection of $93 \%-100 \%$ (see discussion).

\section{$\uparrow \quad$ DISCUSSION}

Fecal GC measures may offer a non-invasive metric for assessing nutritional condition of populations or subpopulations, but not individuals. Our results indicate that fecal GC concentrations vary considerably at given levels of nutritional condition (i.e., percent body fat; Figure 3). The variability of GC across the gradient of nutritional condition we observed is likely due to the overall energetic environment the animal is experiencing, including both endogenous energy reserves and exogenous energy availability (McEwen and Wingfield 2003). Because nutritional stress and GC levels are not expected to rise dramatically until individuals cannot

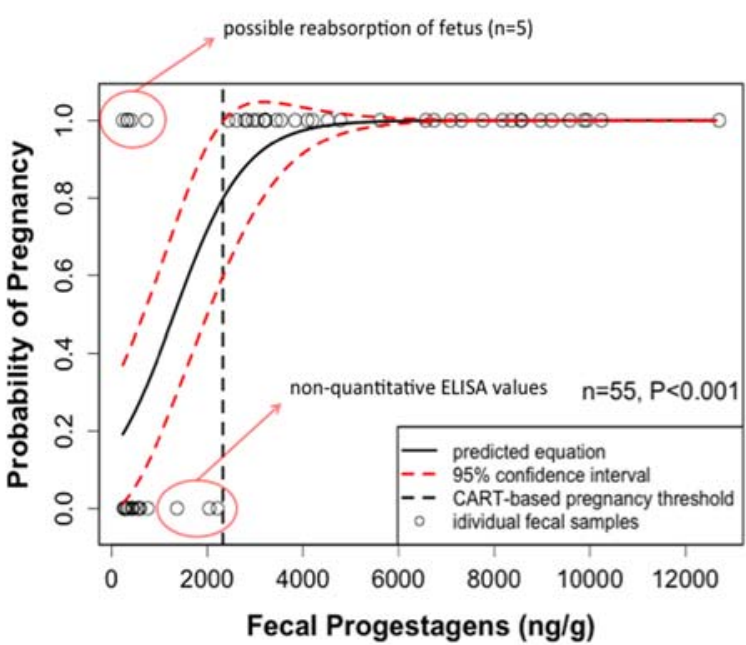

Figure 6. The relationship between fecal progestogen concentrations and pregnancy. Note the 5 false-negatives (upper left corner of plot) and that not all serum samples analyzed for PSPB used quantitative ELISA methods (bottom left corner of plot), which may induce inaccuracy in pregnancy status determination. See discussion for further details.

meet metabolic needs from a combination of both endogenous and exogenous sources, forage quality and availability interact with nutritional condition to determine the GC response (McEwen and Wingfield 2003). Hence, it will be difficult for researchers and managers using fecal-based approaches to disentangle whether elevated GC levels are caused by poor nutritional condition of the animal, deficits in protein or caloric intake, or a combination of these factors. Moreover, large herbivores carry over nutritional reserves from seasonal range to seasonal range (Middleton et al. 2013, Monteith et al. 2013), making the use of GC levels to identify condition of seasonal habitats unreliable. Whereas previous research has demonstrated statistically significant relationships between GC and nutritional condition (e.g., Kitaysky et al. 1999, du Dot et al. 2009, Schultner et al. 2013), our research indicated only weak correlation between $\mathrm{GC}$ levels and nutritional condition (coeff. $=-0.23$, $95 \% \mathrm{CI}=-0.057,0.009)$. Despite the non-significance of our GC model, a more robust sample size will likely yield statistical significance, suggesting that GC levels may serve as a proxy for nutritional condition in comparisons of populations or subpopulations.

Nutritional condition was correlated more strongly with fecal T3 than GC in our study (coeff.= $0.12,95 \% \mathrm{CI}=-0.207,-0.033)$. Similar to the relationship between GC and nutritional condition, T3 varied considerably within individuals in nearly identical nutritional condition (Figure 4). 
Interestingly, the relationship between $\mathrm{T} 3$ and nutritional condition, however strong, did not concur with our biomedical and microcosm-based prediction.

This apparent contradiction has been observed in other free-ranging large mammals (Gobush et al. 2014), and thus deserves further attention. T3 levels were observed to be less variable at given levels of nutritional condition than GC (Figs, 3 and 4). Therefore, once a mechanistic understanding of why T3 levels are inversely related to nutritional condition in large mammals is reached, T3 may serve as a better proxy to population and subpopulation level nutritional condition than GC.

Pregnancy was determined by fecal progestagen concentrations in mid-February (Figure 5). Our results are consistent with previous reports of fecal progestagen thresholds in pregnancy studies of moose (Monfort et al. 1993, Berger et al. 1999, Murray et al. 2012). In this study, CART appears to provide a simple, objective and accurate means of calculating a threshold for fecal pregnancy tests. The CART-based threshold resulted in only a $91 \%(50 / 55)$ accuracy rate, with the 5 misclassified individuals all represented as false-negatives. These 5 misclassifications may have been non pregnant and had aborted fetuses or were in the process of reabsorbing fetuses (Cook et al. 2002), exhibiting low fecal progestagen levels, while ELISA detected the presence of PSPB. Reevaluation of PSPB via quantitative ELISA measures is needed to confirm the $91 \%$ accuracy rate as we would like accuracy to be $>95 \%$. Despite the $<95 \%$ accuracy rate, the reported threshold of $2327.3 \mathrm{ng} / \mathrm{g}$ should perform well for comparing pregnancy rates between populations or subpopulations in nutritional studies and when aiming to assess habitat-performance relationships (Eberhardt 2002, Bowyer et al. 2005).

We have shown evidence that fecal-based measures of $\mathrm{T} 3$ and progestagens provide a noninvasive method for assessing nutritional condition and pregnancy rates in free-ranging populations of moose. Our pilot study was hindered by modest sample sizes and will benefit from augmentation. Despite this limitation, however, we believe that ample evidence has been provided to support continued allocation of time and monies to the validation and refinement of these techniques. Studies occurring in national parks and other settings where capture and handling of animals is undesirable will benefit greatly from the continued pursuit of noninvasive methods for monitoring nutrition and reproduction (Berger et al. 1999). Furthermore, noninvasive tools aimed at aiding our understanding of habitat-performance relationships and life history characteristics will aid researchers and managers in assessment of bottom-up and top-down limitation (Bowyer et al. 2005).

\section{$\downarrow \quad$ LITERATURE CITED}

Bahnak, B. R., J. C. Holland, L. J. Verme, and J. J. Ozoga. 1981. Seasonal and nutritional influences on growth hormone and thyroid activity in white-tailed deer. The Journal of Wildlife Management 45:140.

Berger, J., J. W. Testa, T. Roffe, and S. L. Monfort. 1999. Conservation endocrinology: A noninvasive tool to understand relationships between carnivore colonization and ecological carrying capacity. Conservation Biology 13:980-989.

Bishop, C. J., B. E. Watkins, L. L. Wolfe, D. J. Freddy, and G. C. White. 2009. Evaluating mule deer body condition using serum thyroid hormone concentrations. Journal of Wildlife Management 73:462-467.

Bowyer, R. T., D. K. Person, and B. M. Pierce. 2005. Detecting top-down versus bottom-up regulation of ungulates by large carnivores: Implications for biodiversity. Pages 342-361, In: J. C. Ray, K. H. Redford, R. S. Steneck, and J. Berger (eds.), Large Carnivores and the Conservation of Biodiversity. Island Press, Washington DC, USA.

Breiman, L. 1984. Classification and Regression Trees. CRC Press, Abingdon, UK.

Cain, S. L., M. D. Higgs, T. J. Roffe, S. L. Monfort, and J. Berger. 2012. Using fecal progestagens and logistic regression to enhance pregnancy detection in wild ungulates: A bison case study. Wildlife Society Bulletin 36:631-640.

Clutton-Brock, T. H., S. D. Albon, and F. E. Guinness. 1987. Interactions between population density and maternal characteristics affecting fecundity and juvenile survival in red deer. The Journal of Animal Ecology 56: 857-871.

Cook, J. G., B. Johnson, R. C. Cook, R. A. Riggs, T. Delcurto, L. D. Bryant, and L. L. Irwin. 2004. Effects of summer-autumn nutrition and parturition date on reproduction and survival of elk. Wildlife Monographs 155:1-61.

Cook, R. C., J. G. Cook, R. A. Garrott, L. L. Irwin, and S. L. Monfort. 2002. Effects of diet and body condition on fecal progestagen excretion in elk. Journal of Wildlife Diseases 38:558565 . 
Cook, R. C., J. G. Cook, T. R. Stephenson, W. L. Myers, S. M. Mccorquodale, D. J. Vales, and P. J. Miller. 2010. Revisions of rump fat and body scoring indices for deer, elk, and moose. The Journal of Wildlife Management 74:880-896.

Cook, R. C., T. R. Stephenson, W. L. Myers, J. G. Cook, and L. A. Shipley. 2007. Validating predictive models of nutritional condition for mule deer. Journal of Wildlife Management 71:1934-1943.

Czaplewski, R. L., D. M. Crowe, and L. L. McDonald. 1983. Sample sizes and confidence intervals for wildlife population ratios. Wildlife Society Bulletin 11:121-128.

Danforth, E. 1984. The role of thyroid hormones in the control of energy expenditure. Pages 231239, Vol. 23 In: W. P. T. James (ed.), Clinics in Endocrinology and Metabolism, Obesity, Saunders Co., London, U.K.

Danforth, E., and A. Burger. 1989. The impact of nutrition on thyroid-hormone physiology and action. Annual Review of Nutrition 9:201227.

Du Dot, J. T., D. A. S. Rosen, J. P. Richmond, A. S. Kitaysky, S. A. Zinn, and A. W. Trites. 2009. Changes in glucocorticoids, IGF-I and thyroid hormones as indicators of nutritional stress and subsequent refeeding in Steller sea lions (Eumetopias jubatus). Comparative Biochemistry and Physiology Part A: Molecular and Integrative Physiology 152:524-534.

Eales, J. G. 1988. The influence of nutritional state on thyroid function in various vertebrates. American Zoologist 28:351-362.

Eberhardt, L. L. 2002. A paradigm for population analysis of long-lived vertebrates. Ecology 83:2841-2854.

Gaillard, J. M., M. Festa-Bianchet, N. G. Yoccoz, A. Loison, and C. Toigo. 2000. Temporal variation in fitness components and population dynamics of large herbivores. Annual Review of Ecology and Systematics 31:367-393

Garrott, R. A., S. L. Monfort, P. J. White, K. L. Mashburn, and J. G. Cook. 1998. One-sample pregnancy diagnosis in elk using fecal steroid metabolites. Journal of Wildlife Diseases 34:126-131.

Gobush, K. S., R. K. Booth, and S. K. Wasser. 2014. Validation and application of noninvasive glucocorticoid and thyroid hormone measures in free-ranging Hawaiian monk seals. General and Comparative Endocrinology 195:174-182.
Keech, M. A., R. T. Bowyer, J. M. Ver Hoef, R. D. Boertje, B. W. Dale, and T. R. Stephenson. 2000. Life-history consequences of maternal condition in Alaskan moose. Journal of Wildlife Management 64:450-462.

Kitaysky, A. S., J. C. Wingfield, and J. F. Piatt. 1999. Dynamics of food availability, body condition and physiological stress response in breeding Black-Legged Kittiwakes. Functional Ecology 13:577-584.

Kutner, M. H., and N. Nachtsheim. 2004. Applied Linear Regression Models (Revised Edition with Student CD-ROM.). McGraw-Hill Education.

McEwen, B. S., and J. C. Wingfield. 2003. The concept of allostasis in biology and biomedicine. Hormones and Behavior 43:215.

Middleton, A. D., M. J. Kauffman, D. E. McWhirter, M. D. Jimenez, R. C. Cook, J. G. Cook, S. E. Albeke, et al. 2013. Linking anti-predator behavior to prey demography reveals limited risk effects of an actively hunting large carnivore. Ecology Letters 16:1023-1030.

Monfort, S., C. Schwartz, and S. Wasser. 1993. Monitoring reproduction in captive moose using urinary and fecal steroid metabolites. Journal of Wildlife Management 57:400407.

Monteith, K. L., V. C. Bleich, T. R. Stephenson, B. M. Pierce, M. M. Conner, J. G. Kie, and R. T. Bowyer. 2014. Life-history characteristics of mule deer: Effects of nutrition in a variable environment. Wildlife Monographs, in press.

Monteith, K. L., J. R. Goheen, G. R. Fralick, T. R. Stephenson, and M. J. Kauffman. In Prep. Assessing nutritional condition of Shiras moose: Methodological refinement and implications in a declining population. Canadian Journal of Zoology.

Monteith, K. L., T. R. Stephenson, V. C. Bleich, M. M. Conner, B. M. Pierce, and R. T. Bowyer. 2013. Risk-sensitive allocation in seasonal dynamics of fat and protein reserves in a long-lived mammal. Journal of Animal Ecology 82:377-388

Murray, D. L., K. F. Hussey, L. A. Finnegan, S. J. Lowe, G. N. Price, J. Benson, K. M. Loveless, et al. 2012. Assessment of the status and viability of a population of moose (Alces alces) at its southern range limit in Ontario. Canadian Journal of Zoology 90:422-434. 
Parker, K. L., P. S. Barboza, and M. P. Gillingham. 2009. Nutrition integrates environmental responses of ungulates. Functional Ecology 23:57-69.

Parker, K. L., P. S. Barboza, and T. R. Stephenson. 2005. Protein conservation in female caribou (Rangifer tarandus): Effects of decreasing diet quality during winter. Journal of Mammalogy 86:610-622.

Porterfield, S. P. 1997. Endocrine Physiology. Mosby, Incorporated

Raithel, J. D., M. J. Kauffman, and D. H. Pletscher. 2007. Impact of spatial and temporal variation in calf survival on the growth of elk populations. Journal of Wildlife Management 71:795-803.

Schultner, J., A. S. Kitaysky, J. Welcker, and S. Hatch. 2013. Fat or lean: Adjustment of endogenous energy stores to predictable and unpredictable changes in allostatic load. Functional Ecology 27:45-55.

Stearns, S. C. 1992. The Evolution of Life Histories. Oxford University Press, Oxford UK.
Stephenson, T. R., K. J. Hundertmark, C. C. Schwartz, and V. Van Ballenberghe. 1998. Predicting body fat and body mass in moose with ultrasonography. Canadian Journal of Zoology 76:717-722.

Wasser, S. K., J. C. Azkarate, R. K. Booth, L. Hayward, K. Hunt, K. Ayres, C. Vynne, et al. 2010. Non-invasive measurement of thyroid hormone in feces of a diverse array of avian and mammalian species. General and Comparative Endocrinology 168:1-7.

Wasser, S. K., K. E. Hunt, J. L. Brown, K. Cooper, C. M. Crockett, U. Bechert, J. J. Millspaugh et al. 2000. A generalized fecal glucocorticoid assay for use in a diverse array of nondomestic mammalian and avian species. General and Comparative Endocrinology 120:260-275.

Wikelski, M., and S. J. Cooke. 2006. Conservation physiology. Trends in Ecology \& Evolution 21:38-46. 